\title{
Psychosocial Effects of Teenage Pregnancy: Systematic Analysis
}

\author{
Liranso Gselamu ${ }^{1,}$, , Yoseph Dagne ${ }^{2}$, Martha Gebreyohannes ${ }^{3}$, Andualem Kelebe ${ }^{4}$ \\ ${ }^{1}$ Department of Research and Publication, Yoseph Consultancy, Addis Ababa, Ethiopia \\ ${ }^{2}$ Department of Leadership and Management, Yoseph Consultancy, Addis Ababa, Ethiopia \\ ${ }^{3}$ Department of Public Health, Jimma University, Jimma, Ethiopia \\ ${ }^{4}$ Department of Public Health, Debre Markos University, Markos, Ethiopia
}

Email address:

liranso.2006@gmail.com (L. Gselamu)

${ }^{*}$ Corresponding author

\section{To cite this article:}

Liranso Gselamu, Yoseph Dagne, Martha Gebreyohannes, Andualem Kelebe. Psychosocial Effects of Teenage Pregnancy: Systematic Analysis. Psychology and Behavioral Sciences. Vol. 8, No. 5, 2019, pp. 115-118. doi: 10.11648/j.pbs.20190805.12

Received: May 7, 2019; Accepted: June 25, 2019; Published: October 11, 2019

\begin{abstract}
Globally, teenage pregnancy remains a major problem; but, the available literature does not address this issue properly. The objective of the review was to find out the psychosocial effects of teenage pregnancy. The articles reviewed in this journal were collected from reputable and reliable databases, e-journals and reputed books. The systematic analysis is a form of research that able to address wider issues than single empirical studies ever can [2]. By keeping this in mind, Google Scholar, Databases Scopus, Global Health, Web of Science PubMed, Summon, WHO, PsychInfo, and NIH data sources were used to collect data. English journals/articles, published from 2000 to present were included. To sum up, teenage pregnancy is one psychosocial and economic problems across the globe found in both developed and developing societies. Thus, the effects of teenage pregnancy need further empirical studies since it affects the social-psychological well-being of teenagers and disruption of academic process, school drop-outs, and poor public image.
\end{abstract}

Keywords: Psychosocial, Effects, Teenage, Pregnancy, Teenage Mother

\section{Introduction}

Globally, about 16 million teenage girls' give birth each year [1]. Girls between the ages of 15-19 become pregnant at the early development stage. Girls at this age level are not physically and emotionally fit motherhood responsibilities. It is not the only problem of girls; rather it affects the whole society. For instance, when young girls become pregnant, their health, education, emotions, social life, and future of the society affected at the same time. Its burden affects family lives and exposes them for medical problems and financial instability [2].

It has conclusively been shown that medical problems are a serious issue with its effects. In most developing countries, young girls are not educated on how to care for themselves whilst the pregnancy rate is high. Besides, they are not seeking out adequate medical care during their pregnancy. Many girls are facing difficulties (anemia, toxemia, high blood pressure, placenta previa and premature birth of the baby) during a teen pregnancy [1].

In addition, it has medical effects such as high blood pressure, premature birth, and low baby weight, STDs, postpartum, and loneliness, placenta previa. It is now well established from a variety of studies that teenage pregnancy comes with not only a child but also many consequences. It has higher health risks than mature mothers, such as premature baby, having iron-deficiency, cervical injury, high blood pressure, even death, malnourished and premature delivery [2]. A recent study shows that women aged 15-19 years goes high rate of childbearing [5]. Therefore, the objective of the review was to find out the psychosocial effects of teenage pregnancy.

\section{Method}

The review method was based on the recent research 
literature and relevant information from Global Health, PubMed, Google Scholar, Abstracts, Summon, WHO, and PsychInfo data sources. Numerous journals published 2001 to date were identified and analyzed based on meeting inclusion and exclusion criteria as well as quality standards and analyzed in a systematic manner. Besides, the focus of journals selection was based on the review of the objective which focused on the access to the risk factors associated with teenage pregnancy.

\section{Results and Discussion}

Teenage pregnancy has many life changes. The risk factors associated with adolescent pregnancy [12]. The study findings indicated that the parenting style in the girl's home and teenage pregnancy more common in young women who grow up with permissive and authoritarian parents. Parental relationships with their children are vital. Parents foster open communication in their home, especially around the topics of sex, sexuality, and dating.

However, other researchers found that there was not a relationship between mothers and the effects of sexual media on their daughters' sexual behaviors [15-16]. For example, being pregnant can affect a teenage girl living in education, work freedom, health, loss of friends and families respect.

Culturally, community members, close relatives, friends suddenly isolate and stop socializing pregnant girls. It also exposes for school dropout. Nearly 50 percent of pregnant teens will graduate at the high school level. There is also harsh criticism or scorn from school peers, teachers, and administrators.

Economic challenges, lack of financial support are one of the major problems of a teenage mother in developing countries. In some countries, the law also states that teen mom may attend their normal school based on their preferences. Especially when girls become teenage pregnant, the high school dropout rate is high. Only half of the percentage of teen mothers earns a high school diploma by the time they reach the age of 22 [4].

On the other hand, being pregnant may interfere with work, stress, sleepless nights in arranging child care, and low participation in social activities. It affects prenatal visits and does not take good care of oneself is at greater risk due to labor and delivery complications, anemia, low-birth-weight infant, high blood pressure, fetal death, etc. Likewise, financial problems occur for many teen mothers and affect their children as well. Due to low educational achievement teen mothers getting low income in their lifetime, and nearly one in four will depend on welfare services. Thus, many children will not escape this cycle of poverty. Few of them may complete a high school diploma compared with older parents.

Unwanted pregnancy increases the teenagers stress level, stressful due to education disruptions, being unprepared for parenthood, disruption in their life plans, and financial instability is a major problem among teen mothers. Economically, raising children becoming high prices, there are several necessities to provide a child with the best care including food, strollers, medical insurance, car clothing, seats, etc. Frequently teen moms are unsuccessful to cover the cost of raising a child and getting a decent paying job is even more difficult. Sadly, some babies come with a lot of health issues, not born healthy which makes things very difficult and costly to keep them alive.

A negative consequence for the children of teen mothers mostly costs of teen childbearing associated with, foster care, incarceration, lost tax revenue and increased costs for health care. The probability of help from father for the child's and mother with financial support is low and very difficult for her alone to support the baby. The mother may struggle with rising the child on her own, even two teenagers raising a child is hard [7].

One a study examined the trend mental health of teenage mothers after the pregnancy is completed specifically at depression [7]. They typically exposed for a significantly higher rate of clinical depression both during pregnancy and six months following the birth of their child. Teenage mothers displayed less positive parenting practices and their babies as depression increased and displaying more maladaptive behaviors and the risk of postpartum depression.

Previous studies have explored the relationships between teen pregnancy and poverty $[8,7,3]$. There is a high correlation since teens living in poverty were more likely to get pregnant than teens who do not, their parents often had lower lifetime earnings, as well as social problems and rejections from society. [10] also describes that there is the highest poverty rate among teen's single-parent families, have lower levels of education and have less opportunity in the workplace, and higher stress.

Besides, teenage women who become pregnant get blamed for causing adult poverty, frequently criticized their children and welfare dependence, and as a social problems, absence of reliable child care, inadequate housing, and the lack of health care. There is a relationship between risk behaviors (drinking, smoking, and drugs) and the likelihood of becoming pregnant [8]. But it is hard to provide full and reliable evidence that certain behaviors make teenage women more likely to become pregnant, frequently those who are least well-prepared to nurture a child are most likely to become pregnant.

Eventually, adolescent mothers have become increasingly likely to remain single parents, and thus, some researchers argue that the discrepancies between the realities of teen pregnancy and the strategies for its causes and prevention. The author believed this issue is due to lack of sexual enlightened from parents and schools. For instance, most of the developing countries cannot decrease sexual engagement due to poor educational systems and culture. Education policies should including sex education program as part of the curricula and improving adolescents' healthy life as an investment in further education.

Moreover, unwanted pregnancy and early sexual activity may lead to serious maternal and fetal complications, thus, placing the individual as the main decision maker regarding 
the social determinants of sexual health. Risk behaviors/predisposing factors include drug addiction, sexual promiscuity, alcoholism, social and health problems among adolescents and sexual abuses resulting in an unwanted pregnancy [8]. There is a relationship between risk behaviors and pregnancy among teenagers. Most of these girls are victims due to lack information; schools could have enabled them to deal with friends who lure them into sex prematurely and about the causes and consequences of teen pregnancy and childbearing.

The effect of teenage pregnancy could be devastating since it is not only on the teenage-mother, it also continues with their education and social life. For example, teenage-mother may fear to express that eventually gets married and decreases her education drastically; she becomes the primary housewife and faces economic challenges. She may face higher risks which usually plagued by intellectual language and socio-emotional delays and hardships which extends beyond birth and problems in cognition, language communication, and interpersonal skills. It also exposes for regret and disappointment, emotional trauma they went through while the pregnancy lasted, even after delivery and psychological problems. Another painful experience is the rejection by the male counterparts, by relatives and peers decrease closeness.

In fact, pregnancy at whatever stage in life can be a life changing experience cuts educational attainment and socioeconomic status, stress, dislike, malice, boredom, and unhappiness experienced by a teenage girl within her home environment. Frequently, children of single parents are more vulnerable to teenage pregnancy and exposure to most sexual content on television, sexuality in the media and pornographic and sex chat rooms by teenagers and mainly tuned to engage in sexual activities.

To sum up, teenage pregnancy is one psychosocial and economic problems across the globe found in both developed and developing societies. Thus, the effects of teenage pregnancy need further empirical studies since it affects social-psychological the well-being of teenagers and the disruption of the academic process, school drop-outs and poor public image.

\section{Conclusion}

In general, therefore, it seems that the teenage pregnancy rate is still high but the available literature does not address it well. School environments should be protective enough and teachers and parents should empower children on negative influences, the well-being of the girl child through education about sex and sexuality. Awareness creation of the girl child should be made free and compulsory at their understanding level. On the other hand, the stress associated with pregnancy, sense of rejection by friends of relatives, lowered self depression and emotional trauma, fear of future and deprivation usually challenges faced by teenage mothers which may exposes to mental illness. Suddenly being unprepared for parenthood, sudden monetary burden, realization that the teen will have a lifelong connection with the other parent or ending the relationship with the other parent, disruption in their life plans, and increases the teenagers' stress level. Local government offices and social welfare agencies should be involved in supporting and providing professional and legal services, counseling and rehabilitation of victims engage in other meaningful activities to avoid early destitution It does not only do affect teen mothers; it also affects the immediate family and community at large. Stakeholders (school administrators, teachers, adults, and counselors) working with teenagers ma play a role in minimizing these risks. The stakeholder can offer to educate, mentor, and create policies that can have a positive impact on teenagers' lives.

\section{References}

[1] Alice, S. (2012). Teen pregnancy, International journal of adolescence and youth. Early child development and care, NY, USA.

[2] Baumeister, R. F. (2013). Writing a literature review. In M. J. Prinstein \& M. D. Patterson (Eds.), the portable mentor: Expert guide to a successful career in psychology (pp. 119132; 2nd ed.). New York: Springer Science + Business Media.

[3] Bissell, M. (2000). Socio-economic outcomes of teen pregnancy and parenthood: A review of the literature. Canadian Journal of Human Sexuality, 9 (3), 191-105.

[4] Centers for Disease Control (2019). Reproductive Health: Teen Pregnancy. Atlanta, Georgia.

[5] CSA (2016). Ethiopia Demographic and Health Survey Addis Ababa, Ethiopia.

[6] Culp, R. (2012). Teen Pregnancy Negatively Impacts the National Economy. Think Progress RSS.

[7] National Campaign to Prevent Teen Pregnancy (NCPTP). (2002). Not just another single issue: Teen pregnancy prevention's link to other critical issues.

[8] National Council of Welfare. (2004). The cost of poverty (Winter 2001-02). National Council of Welfare, Ottawa.

[9] Sprague, C. (2009). Teen Pregnancy. Research Starters Sociology (Online Edition) Research Starters.

[10] Sawhill, I. (2001). What can be done to reduce teen pregnancy and out-of-wedlock births? Brookings Policy

[11] Thikra, N., Khawla, I., Ulfat, M., Yousif, A. (2015). The Impact of Teenage Pregnancy on Maternal, Fetal and Neonatal Outcomes. International Journal of Scientific Research in Knowledge, 3 (4), pp. 0106-113.

[12] VanDyk, G. (2011). Risk factors associated with adolescent pregnancy; An exploratory study. Journal of Psychology in Africa, 21 (4), 581-284.

[13] Waltohar Single (2019). Mothers: The Effects of Teen Pregnancy on Children: Articles for Single Parents." Single Mothers: The Effects of Teen Pregnancy on Children: Articles for Single Parents. N. p. 
[14] WHO. (2014). Adolescent pregnancy fact sheet. WHO, Geneva.

[15] World Health Organization (2019). Adolescent Pregnancy. WHO, Geneva.
[16] Wright, P., Randall, A., \& Arroyo, A. (2013). Father-daughter communication about sex moderates the association between exposure to MTV's 16 and Pregnant/Teen Mom and female students' pregnancy-risk behavior. Sexuality \& Culture, 17 (1), 50-66. 